\title{
The use of the ensemble Kalman filter for production management in the presence of impermeable obstructions
}

\author{
George Saad ${ }^{1} \cdot$ Fouad Azizi $^{2}$
}

Received: 1 June 2015/Accepted: 25 October 2015/Published online: 18 November 2015

(C) The Author(s) 2015. This article is published with open access at Springerlink.com

\begin{abstract}
This study presents a novel approach for maximizing the efficiency of the water flooding process by controlling the flood front dynamics. The optimization is carried out assuming the presence of spatially varying geological properties and impermeable obstructions located between the injection and production wells and thus hindering the sweep efficiency inside the reservoir. Controlling the flood front dynamics is achieved by coupling an ensemble Kalman filter scheme with a two-phase immiscible flow reservoir simulator and thus relying on a set of observational data provided via a grid of smart wells. For that purpose, a virtual grid of smart wells assumed to be fully saturated with water and evenly distributed around the obstruction location is employed. The presented method is applied to a synthetic numerical example where the fluid front is tracked to maximize water flooding by controlling the injection and production rates.
\end{abstract}

Keywords Closed loop reservoir management . Ensemble Kalman filter · Reservoir simulation .

Water-flooding

\section{List of symbols}

CLRM Closed loop reservoir management (-)

COP Cumulative oil production $\left(\mathrm{m}^{3}\right)$

$d \quad$ Distance to centerline of obstruction (m)

George Saad

george.saad@aub.edu.lb

1 Department of Civil and Environmental Engineering, American University of Beirut, Riad El Solh, P.O. Box 11-0236, Beirut 1107 2020, Lebanon

2 Department of Chemical Engineering, American University of Beirut, Beirut, Lebanon
D Ensemble of observations (-)

EnKF Ensemble Kalman filter (-)

$f \quad$ Frequency of updates (days)

$H \quad$ Observation matrix (-)

$h_{1} \quad$ Distance between injection wells (m)

$h_{2} \quad$ Height of obstruction (m)

$K \quad$ Intrinsic permeability (Darcy)

$K_{\text {ro }} \quad$ Oil relative permeability (-)

$K_{\text {rw }} \quad$ Water relative permeability (-)

$K_{t} \quad$ Kalman gain computed at time $t(-)$

NPV Net present value (\$)

$p_{\mathrm{c}} \quad$ Capillary pressure $(\mathrm{Pa})$

$P_{\mathrm{e}} \quad$ Model error covariance matrix (-)

$p_{\mathrm{o}} \quad$ Oil pressure $(\mathrm{Pa})$

$p_{\mathrm{w}} \quad$ Water pressure $(\mathrm{Pa})$

$Q \quad$ Vector containing flow rates $\left(\mathrm{m}^{3} /\right.$ day)

$q_{\mathrm{o}} \quad$ Oil production rate $\left(\mathrm{m}^{3} /\right.$ day $)$

$q_{\mathrm{w}} \quad$ Water injection rate $\left(\mathrm{m}^{3} /\right.$ day $)$

$R_{\mathrm{e}} \quad$ Measurement error covariance matrix (-)

$S_{\mathrm{e}} \quad$ Reduced water saturation (-)

$S_{\mathrm{m}} \quad$ Maximum water saturation (-)

$S_{\mathrm{o}} \quad$ Oil saturation (-)

$S_{\mathrm{w}} \quad$ Water saturation (-)

$S_{\mathrm{wc}} \quad$ Critical water saturation (-)

$U \quad$ Matrix holding ensemble of state vectors (-)

$V \quad$ Vector containing saturation profile (-)

$X \quad$ Length of the reservoir (m)

$Y \quad$ Height of the reservoir (m)

\section{Greek symbols}

$\mu_{\mathrm{o}} \quad$ Oil viscosity (Pa s)

$\mu_{\mathrm{w}}$ Water viscosity (Pa s)

$\phi \quad$ Porosity (-) 


\section{Background}

The hydrocarbon production process goes through several stages. In the primary recovery stage, the natural reservoir energy displaces hydrocarbons from the reservoir, into the wellbore and up to surface. This stage reaches its limit once the reservoir pressure is too low that the production rate is not economical. In the second recovery stage, fluids such as gas or water are injected through injection wells to help maintain the pressure of the reservoir. When water is used, the process is commonly known as water flooding. The injected water physically sweeps the displaced oil and eventually reaches the production wells. The percentage of water in the produced fluids steadily increases until the cost of removing and disposing of water exceeds the income from oil production. At that stage, more sophisticated techniques that alter the original properties of the reservoir are used. These enhanced recovery techniques involve expensive chemical and thermal processes.

Whereas the primary recovery process is limited by the reservoir properties and characteristics (e.g., pressure and depth), minimizing the costs associated with the enhanced recovery techniques, is tantamount to optimizing the secondary recovery stage. While water flooding is the most commonly used method for secondary oil recovery, there exist potential problems associated with it such as its inefficiency due to variable permeability or other conditions affecting fluid transport within the reservoir. These conditions could be pertaining to an impermeable obstruction where oil might migrate and get trapped below or within such obstacles.

The design of an economically optimal recovery strategy is usually based on reservoir simulation, i.e., large-scale numerical simulation of the flow of multi-phase fluids through strongly heterogeneous porous media (Jansen 2011). The efficiency of a reservoir model relies on its ability to characterize the geological and petrophysical features of the actual field. One of the most commonly used methods for reservoir characterization is the automatic history matching methodology. This is a type of inverse problem that aims at estimating reservoir model parameters such as porosities and permeabilities, among others, so as to minimize the mismatch between actual measurements and simulated values (Gu and Oliver 2006; Oliver and Chen 2011; Saad and Ghanem 2009). There are several approaches for automatic history matching which differ in whether the error function used to optimize the model parameters is linear or nonlinear (Krymskaya et al. 2009). Most of the traditional history matching techniques do not allow for continuous model updating, instead they simultaneously use all recorded data to generate an appropriate reservoir flow model. However, with the recent technological advances and the deployment of permanent sensors to monitor the various reservoir and production parameters, the use of continuously available data becomes critical for keeping the reservoir model up-todate (Bianco et al. 2007; Gu and Oliver 2006; Krymskaya et al. 2009). Therefore, to overcome some of the limitations associated with traditional history matching techniques, sequential data assimilation methods are required. One of the most commonly used methods is the ensemble Kalman filter (EnKF), which is a Monte Carlo based methodology for history matching and real time updates of reservoir models (Aanonsen et al. 2009; Chen et al. 2009; Gu and Oliver 2006; Krymskaya et al. 2009; Lorentzen et al. 2006; Oliver and Chen 2011). First introduced by Evensen (1994) to calibrate oceanographic models (Bianco et al. 2007), the EnKF has since been successfully employed in both groundwater and reservoir simulation and several review articles that assess its performance and successes are available (Aanonsen et al. 2009; Evensen 2003; Oliver and Chen 2011). It was generally found to provide good history matching and useful estimation of various reservoir parameters while requiring less computation work as compared to other techniques (Aanonsen et al. 2009; Gu and Oliver 2006).

Combining data assimilation with production optimization results in what is known as the closed loop reservoir management (CLRM), where the objective of production optimization is to determine optimum operating strategy to maximize a desired outcome, such as net present value (NPV), or the cumulative oil production (COP). Production optimization has been gaining momentum in upstream petroleum industry as it allows maintaining the production rate of the reservoir over a longer period of time, and therefore increasing oil production at a reduced cost (Asadollahi et al. 2014; Dehdari and Oliver 2012). Several optimization tools are employed for this purpose. While most of them are gradient based methods (Asheim 1988; Brouwer et al. 2004; Nævdal et al. 2006; Sarma et al. 2006; van Essen et al. 2009), other techniques such as genetic algorithms, simulated annealing, SQP, and SPSA, are also available. These methods are presented and thoroughly discussed and compared in various works (Asadollahi et al. 2014; Dehdari and Oliver 2012; Fonseca et al. 2014; Wang et al. 2009).

While the EnKF is the most commonly used data assimilation technique for history matching, it is rarely used for the purpose of production optimization. This has been attributed to the inherent nature of the method where a large number of iterations is required, rendering it more time consuming when compared to others (Lorentzen et al. 2006; Wang et al. 2009). However, the fact that its implementation is easier than other methods is often overlooked. For example, the implementation of the adjoint 
methods require detailed information about the reservoir simulator (Wang et al. 2009), or it is not always possible to obtain an accurate gradient from commercial simulators, or sometimes the objective function is not differentiable (Dehdari and Oliver 2012). To the authors' knowledge, the use of the EnKF for CLRM has been limited to only a few studies. Lorentzen et al. (2006) were the first to propose its usage for production optimization by controlling downhole chokes. They compared this method to the partial enumeration method and concluded that while the EnKF is more time consuming, it produces better results in terms of optimal oil production and optimal water injection and production. Their work was followed by Nwaozo (2006) who extended their concept and used an average gradient from the ensemble of realizations, and reported a remarkable improvement in NPV and COP. Wang et al. (2009) also used the EnKF for reservoir model updating and compared three techniques for production optimization, namely, EnKF, SPSA, and steepest ascent. Nonetheless, they concluded that the steepest ascent method is the most efficient while the EnKF, when treated as a production optimization algorithm, yielded poor estimates of the optimal controls.

Thus far, all previous studies that employed the EnKF for production optimization in CLRM did not investigate the effect of the optimization model parameters on the outcome. The present study will focus on optimizing the front dynamics in the presence of obstructions that might hinder the flow. In particular, it will focus on the case where the presence of an impermeable obstacle between the injection and production wells hinders the sweep efficiency inside the reservoir. Accordingly, the effect of number of ensembles, frequency of updates, aspect ratio of the obstacle, shape of the obstacle, and the distance between injection/production wells and the obstacle, on the sweep efficiency behind the obstacle will be investigated. In cases where the injected fluid composition is fixed, the only given way to control the front dynamics is therefore through controlling the allocation of the injected fluid to the injection wells.

\section{Reservoir simulation model}

Reservoir simulation is a powerful tool for reservoir characterization and management as it enhances the production forecasting process. In this study, a finite volume MATLAB simulator is developed to model the reservoir multi-fluid phase dynamics. The simulator is designed to provide the information pertaining to the velocity, pressure, temperature, and saturation of the various phases flowing within the medium.
The problem at hand is a two-phase immiscible flow problem. Initially the porous medium is assumed to be fully saturated with oil, and then water is pumped through injection wells to push the oil out through other wells in the field. To model this multiphase flow system, conservation of mass for each existing phase is required. The governing flow equations consist of the water continuity equation,

$\phi\left(\frac{\partial S_{\mathrm{w}}}{\partial t}\right)=\nabla \cdot\left[\frac{\vec{K} K_{\mathrm{rw}}}{\mu_{\mathrm{w}}}\left(\nabla P_{\mathrm{w}}-\rho_{\mathrm{w}} g \nabla z(x)\right)\right]+q_{\mathrm{w}}$

and the oil continuity equation,

$\phi\left(\frac{\partial S_{\mathrm{o}}}{\partial t}\right)=\nabla \cdot\left[\frac{\vec{K} K_{\mathrm{ro}}}{\mu_{\mathrm{o}}}\left(\nabla P_{\mathrm{o}}-\rho_{\mathrm{o}} g \nabla z(x)\right)\right]+q_{\mathrm{o}}$

where $\phi$ is the porosity of the medium, $S_{\mathrm{w}}$ and $S_{\mathrm{o}}$ denote the water and oil saturations, $t$ the time, $\vec{K}$ the intrinsic permeability, $K_{\mathrm{rw}}$ and $K_{\text {ro }}$ the relative permeability of water and oil, $\mu_{\mathrm{w}}$ and $\mu_{\mathrm{o}}$ represent viscosities, $P_{\mathrm{w}}$ and $P_{\mathrm{o}}$ the water and oil pressures, $g$ the gravitational acceleration, $z$ the depth of the fluid, and $q_{\mathrm{w}}$ and $q_{\mathrm{o}}$ are the source/sink terms. The above equations are subject to the following constraints:

$S_{\mathrm{W}}+S_{\mathrm{o}}=1$

$p_{\mathrm{c}}\left(S_{\mathrm{w}}\right)=P_{\mathrm{o}}-P_{\mathrm{w}}$

where $p_{\mathrm{c}}$ is the capillary pressure representing the pressure difference that occur across the fluid-fluid interfaces.

The latter set of equations signifies that the reservoir flow is driven by gravity, pressure gradients, and viscous forces. It also incorporates the effects of porous matrix compressibility, fluid compressibility, capillary pressure, and spatial variability of permeability and porosity. The nonlinearity arises from the constitutive relations relating the phase relative permeabilities and capillary pressure to the phase saturations. In this study, the Brooks-Corey empirical model Brooks and Corey (1964) is used to define these relationships in the form,

$K_{\mathrm{rw}}=S_{\mathrm{e}}^{(2+3 \lambda) / \lambda}$

and

$K_{\mathrm{ro}}=\left(1-S_{\mathrm{e}}\right)^{2}\left(1-S_{\mathrm{e}}^{(2+\lambda) / \lambda}\right)$

where $\lambda$ is a model fitting parameter related to the pore size distribution of the soil material, and $S_{\mathrm{e}}$ is the reduced saturation given by

$S_{\mathrm{e}}=\frac{S_{\mathrm{w}}-S_{\mathrm{wc}}}{S_{\mathrm{m}}-S_{\mathrm{wc}}}$

where $S_{\mathrm{m}}$ is the maximum water phase saturation and $S_{\mathrm{wc}}$ is the critical saturation at which the injected water starts to 
flow. Brooks and Corey's (1964) equation for the capillary pressure is,

$p_{\mathrm{c}}=p_{\mathrm{d}} S_{\mathrm{e}}^{-1 / \lambda}$

where $p_{\mathrm{d}}$ is the displacement or threshold pressure which first gives rise to the oil phase permeability.

The reservoir model is then coupled with a nonlinear sequential data assimilation scheme for tracking the fluid front dynamics to maximize the water flood sweeping efficiency by controlling the injection rates.

\section{Sequential data assimilation}

Data assimilation is a versatile methodology for estimating unknown state variables and parameters. It relies on a set of observational data and the underlying dynamical principles governing the system under observation. General schemes for data assimilation often relate to either estimation theory or control theory, but some approaches like direct minimization, stochastic and hybrid methods can be used in both frameworks.

In estimation theory, statistical approaches are used to estimate the state of a dynamical system by combining all available knowledge pertaining to the system including the measurements and the modeling theories. Of significant importance in the estimation process is the a priori hypotheses and melding criterion since they determine the influence of dynamics and data onto the state estimate. One of the most widely used tools in estimation theory is the Kalman filter (Kalman 1960), which gives a sequential, unbiased, minimum error variance estimate based upon a linear combination of real time observations and dynamics. The Kalman filter is an optimal sequential data assimilation method for linear dynamics and measurement processes with Gaussian error statistics. It provides a linear, unbiased, minimum variance algorithm to optimally estimate the state of the system from noisy measurements. The Kalman filter loses its optimality for nonlinear dynamical systems. As such many extensions of the Kalman Filter have been developed to tackle the different challenges associated with the problem of sequential data assimilation. In this study the EnKF will be used for optimizing the water flooding sweep efficiency.

\section{The ensemble Kalman filter}

The EnKF proposed by Evensen (1994) and expanded by Burgers et al. (1998) is based on forecasting the error statistics using Monte Carlo sampling. The EnKF propagates an ensemble of state vectors forward in time and updates the state vectors as measurements become available. Assuming the reservoir petrophysical and geological properties are known, the EnKF in this study is used for production optimization to maximize the sweep efficiency of the water flooding process. As such, the model state vector, $U$, is augmented to include the dynamic state variables and the time varying water injection and oil production rates:

$U=\left[\begin{array}{ll}V & Q\end{array}\right]^{\mathrm{T}}$

where $V$ consists of the water saturation for each grid block in the numerical solution scheme and $Q$ represents the respective water injection and oil production rates. The initial ensemble is chosen so that it properly represents the error statistics of the initial guess of the model states. Therefore, the initial ensemble is usually created by adding some kind of perturbations to a best-guess estimate, and then the ensemble is integrated over a time interval covering a few characteristic time scales of the dynamical system.

Let $U$ be the matrix holding the ensemble members $U_{i} \in R^{n}$,

$U=\left(U_{1}, U_{2}, \ldots, U_{N}\right) \in R^{n \times N}$

where $N$ is the number of ensemble members, and $n$ is the size of the model state vector. The ensemble mean is given by,

$\bar{U}=U 1_{N}$

where $1_{N} \in R^{N \times N}$ is a matrix with all its elements equal to $1 / N$. The model error covariance matrix can be computed as,

$P_{\mathrm{e}}=\frac{(U-\bar{U})(U-\bar{U})^{\mathrm{T}}}{N-1}$

The EnKF algorithm consists of two steps, a forecast step $\left(U^{\mathrm{f}}\right)$ and an update step $\left(U^{\mathrm{a}}\right)$. The forecast step is calculated by using the reservoir simulator to propagate the ensemble of state vectors from time step $(t-1)$ to time step, $t$,

$U_{t, i}^{\mathrm{f}}=f\left(U_{t-1, i}^{\mathrm{a}}\right)+\xi_{i} \quad i=1, \ldots, N$

where the set of $\varepsilon_{i}$ 's is that of independent, identical, zero mean perturbations having a covariance representing the model error associated with the mathematical model uncertainty. In the update step, the ensemble of forecasted state vectors $U_{t}^{\mathrm{f}}$ is updated to minimize the mismatch between the measurements and the corresponding predictions from the simulator. An ensemble of observations is generated by adding perturbations with zero mean and covariance equal to the measurement error covariance matrix to the true observation vector at time, $t$ (Burgers et al. 1998). The ensemble of state vectors is related to the measurement variables via an observation matrix, $H$ : 
$d_{i}=H U_{t}+\epsilon_{i} \quad i=1, \ldots, m$

where $H U_{t} \in R^{m}$ is the true observation vector corresponding to selected measured values in the true state vector, $U_{t}, m$ is the number of measurements, and the set of $\varepsilon_{i}$ is that of independent, identical, zero mean perturbations having a covariance representing the measurement error.

Let $D$ be the matrix holding the ensemble of measurements $d_{i} \in R^{m}$ :

$D=\left(d_{1}, d_{2}, \ldots, d_{N}\right) \in R^{m \times N}$

The corresponding measurement error covariance matrix is given by,

$R_{\mathrm{e}}=\frac{\gamma \gamma^{\mathrm{T}}}{N-1}$

where $\gamma=\left(\epsilon_{1}, \epsilon_{2}, \ldots, \epsilon_{N}\right) \in R^{m \times N}$ is the ensemble of measurement perturbations. The updated states are computed as,

$U_{t, i}^{\mathrm{a}}=U_{t, i}^{\mathrm{f}}+K_{t}\left(d_{t, i}-H U_{t, i}^{\mathrm{f}}\right)$

where $K_{t}$ is called the Kalman gain matrix and is given by,

$K_{t}=P_{\mathrm{e}} H^{\mathrm{T}}\left(H P_{\mathrm{e}} H^{\mathrm{T}}+R_{\mathrm{e}}\right)^{-1}$.

\section{Optimization approach}

The optimization approach adopted for solving this problem is presented in flow chart form in Fig. 1. Starting with a characterized reservoir model; i.e., a model with known geological and petrophysical properties and a grid of smart wells that provide measurements at a known frequency, and given a water injection rate, the reservoir simulator is used to propagate the system forward in time. Whenever measurements are available the EnKF is employed to optimize the injection rates so as to minimize the mismatch between the model prediction and the desired values of control variables assigned as elements in the observation vector. These control variables represent the saturation profiles at selected measurement locations.

The main objective of this study is to optimize the front dynamics in the presence of spatially varying permeabilities and obstructions that might hinder the flow. Therefore, a virtual grid of 81 smart wells assumed to be fully saturated with water and evenly distributed around the obstruction location is employed for the optimization scheme. Full water saturation was assumed when water fills $85 \%$ of the pore, i.e., $S_{\mathrm{w}}=0.85$, and the wells were systematically placed with 54 wells upstream of the obstacle and 27 of them downstream of the obstacle as shown in Fig. 2. The optimized water flooding process will therefore delay the water breakthrough and improve the sweep efficiency in the region upstream of the non-permeable region, and every optimization run will stop once the injected water reaches the production wells. However, the presented optimization scheme is limited to only optimizing well flow rates and does not include well locations or configurations as control variables to optimize the production.

\section{Numerical application}

The problem at hand is illustrated using a 2D example that involves two injectors and two producers as shown in Fig. 3. The reservoir dimensions are $1,400 \times 400 \times 10 \mathrm{~m}$, and the porosity is represented as a random process drawn from a Gaussian distribution with a mean of 0.35 and a SD of 0.05 . The spatial distribution of the porosity in the reservoir is depicted in Fig. 4. The reservoir permeability is assumed to be constant, $K=1,000 \mathrm{md}$, throughout the medium except for the highlighted impermeable zones depicted in Fig. 3, where the permeability was set equal to zero $(K=0 \mathrm{md})$. The oil and water viscosities are assumed to be $10^{-2}$ and $10^{-3} \mathrm{~kg} / \mathrm{m} \mathrm{s}$, respectively. Furthermore, the reservoir is initially assumed to be fully saturated with oil. For the purpose of this study, a pore is assumed to be fully saturated with oil when $S_{\mathrm{o}}=0.8$ (allowing a minimum water content in every pore, with $S_{\mathrm{w}}=0.2$ ), and fully saturated with water when $S_{\mathrm{w}}=0.85$, i.e., $S_{\mathrm{o}}=0.15$.

As a base case, an equal volumetric flow rate of $65 \mathrm{~m}^{3} /$ day was allotted to each injector, the total water injection rate therefore sums up to $130 \mathrm{~m}^{3} /$ day for both wells. The optimization is then performed on both the injection and production rates to delay the water breakthrough and improve the sweep efficiency of the oil in the region upstream of the obstacle.

Within the framework of the EnKF, an ensemble of state vectors is used to represent the uncertainty within the system. Sensitivity analysis is conducted to optimally select the number of ensembles to be used for the model parameter calibration process. Based on the sensitivity analysis results, it was decided to use an ensemble size of 60 for adequate representation of the system noise. The initial ensemble is propagated forward in time using the reservoir simulator. The model error is represented via an additive Gaussian white noise having a SD equal to $10 \%$ of the forecasted state vectors. Whenever measurements are available, the model state vectors are updated using the Kalman filter equations. The measurement errors are represented via an additive Gaussian white noise having a SD equal to $5 \%$ of the measured reading. During optimization, 
Fig. 1 Front control flow chart

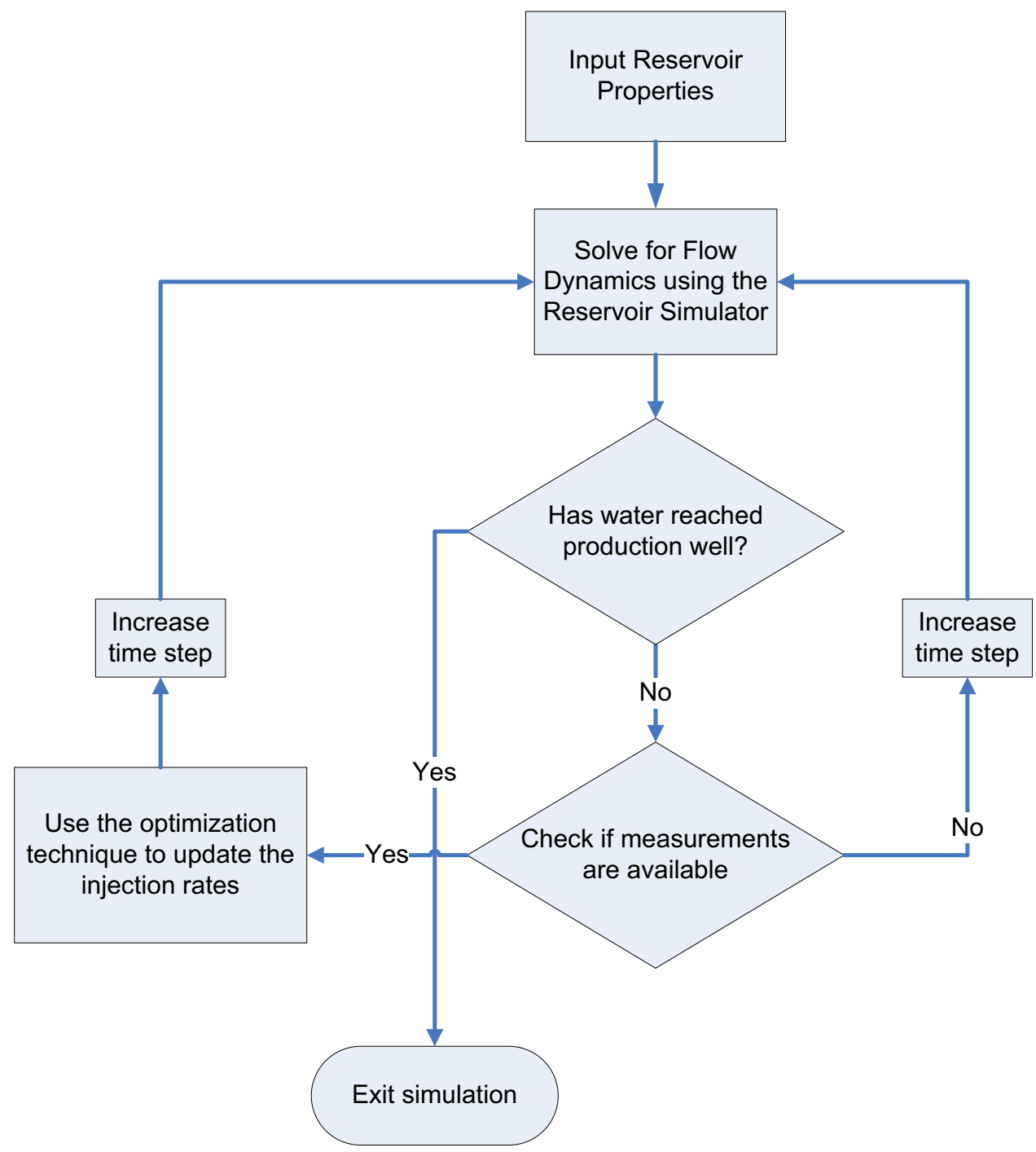

the volumetric flow rates for the injection and production were controlled by the EnKF with the imposed constraint of a maximum allowable injection/production rate per well set at $130 \mathrm{~m}^{3} /$ day. After each update, checks are carried out to verify that the updated water saturation values are physical, i.e., they fall within the bounds between 0.2 and 0.85 . Whenever the water saturation values exceeded the pre-set bounds, a truncation measure was applied to set them at the respective limits.

Additionally, two types of obstructions were considered, namely, rectangular and U-shaped as shown in Fig. 3a and b. In this work, sensitivity analyses to investigate the effects of the size, shape and location of the obstruction on the water flooding sweep efficiency were conducted. The size and location effects were studied by varying the aspect ratio, $h_{2} / h_{1}$, and the distance of the obstruction away from the reservoir boundaries, $d$, respectively. Furthermore, the effect of the frequency of updates, $f$, was also investigated.

\section{Example}

As previously mentioned, the reservoir is initially assumed to be fully saturated with oil $\left(S_{\mathrm{o}}=0.8, S_{\mathrm{w}}=0.2\right)$, then at time $t=0$, water starts flowing from the injection wells. Figure 5 below presents snap shots of the water saturation profiles at different stages of the water flooding process. These snap shots correspond to the case of a rectangular obstruction (20 m thick) located at a distance $d=200 \mathrm{~m}$ (corresponding to a $d / X=2 / 14$ ) and having an aspect ratio $\left(h_{2} / Y\right)$ of 0.375 . The optimization is carried out assuming that injection and production rates are updated every 15 days ( $f=15$ days). The sweep efficiencies upstream of the non-permeable region are reported for both the optimized and base cases at different time stages leading to breakthrough. The sweep efficiency is calculated as the percent sweep in all the cells located directly upstream of the obstruction using the following equation: 


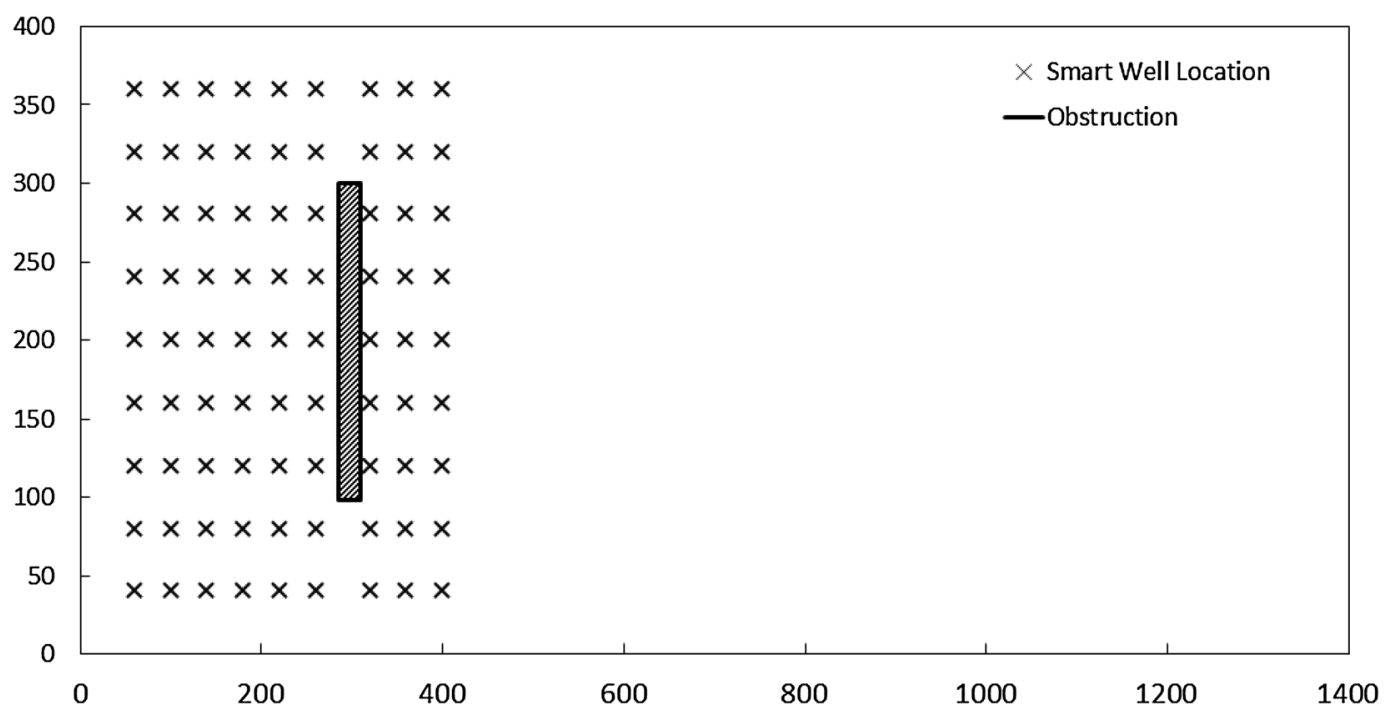

Fig. 2 Location of the smart wells with respect to the non-permeable location (obstacle centerline located at $x=300$ )

Fig. 3 Schematic representation of the reservoir geometry considered in the two studies a rectangular obstruction; b U-shaped obstruction
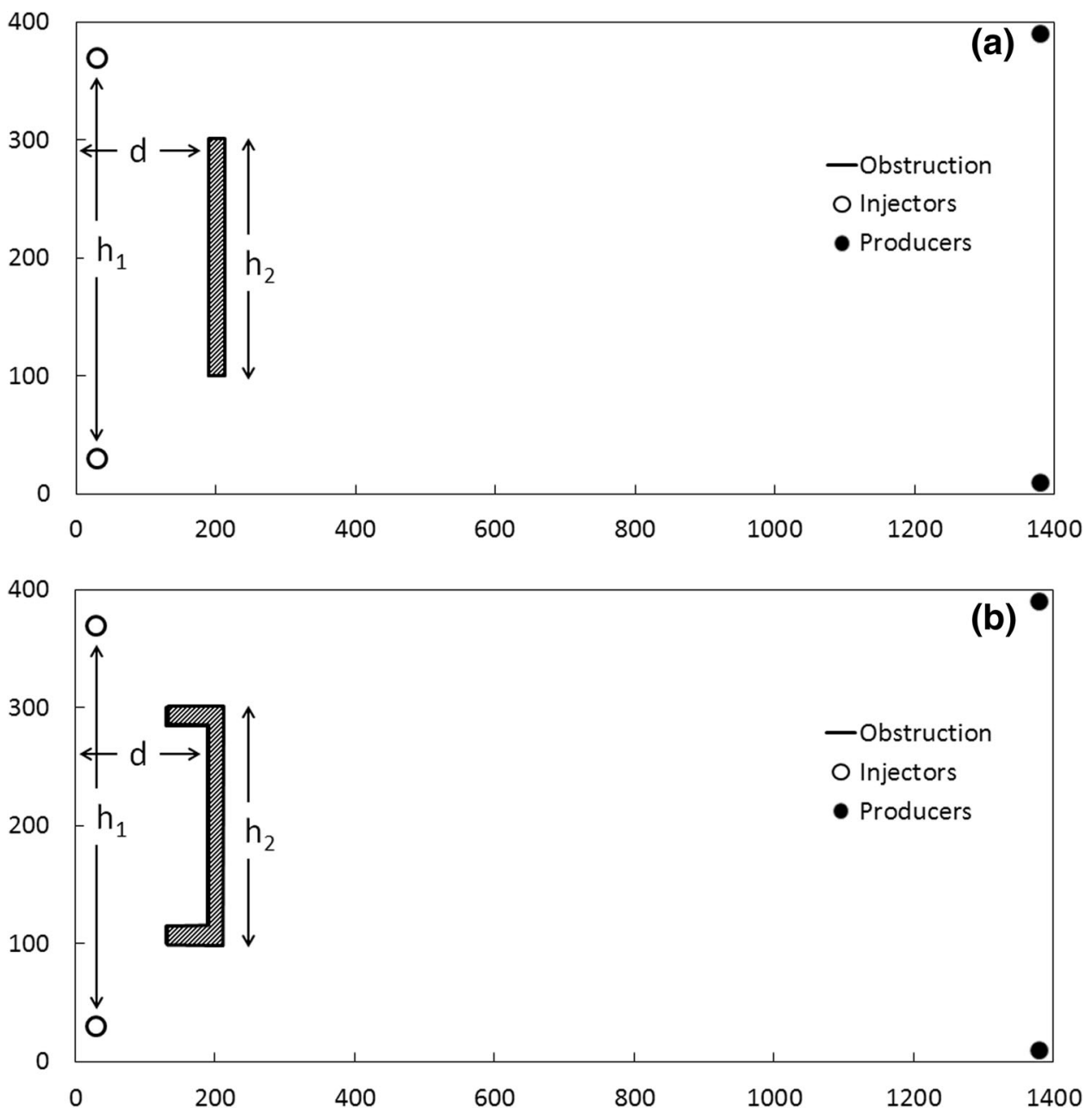


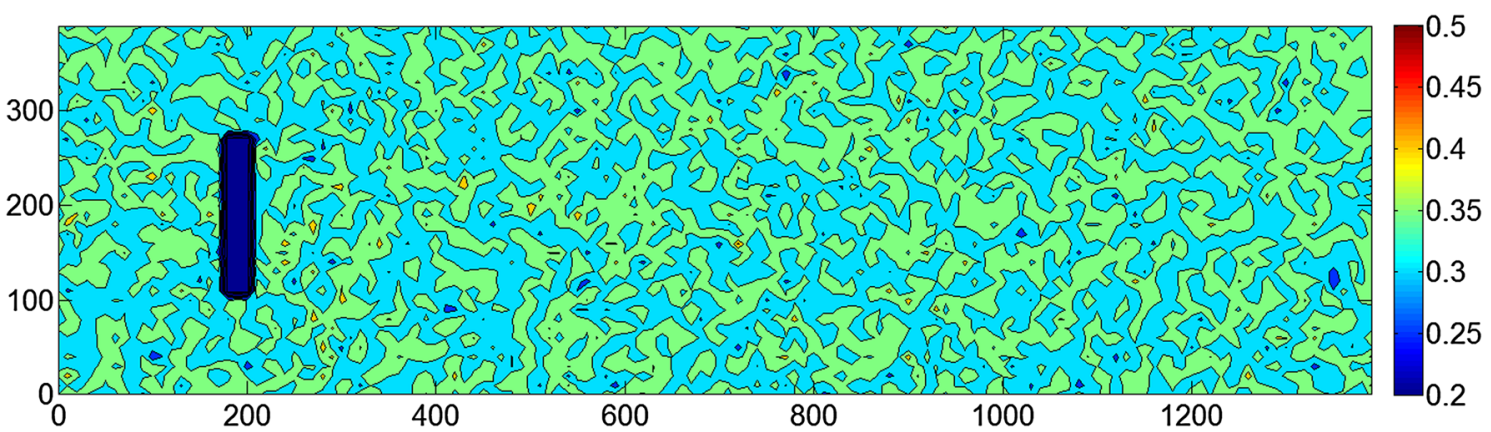

Fig. 4 Spatial distribution of the porosity in the medium

\section{Optimized}

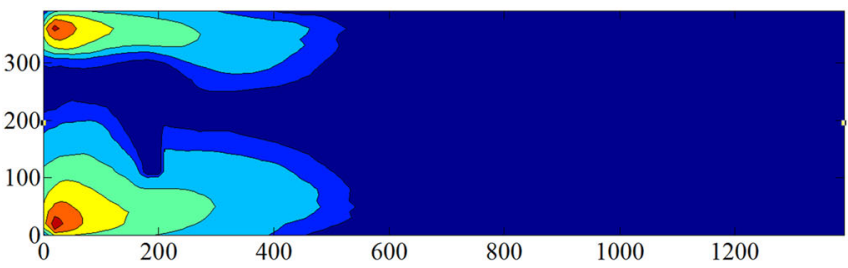

$\mathrm{t}=100$ days - Sweep Efficiency $=20.58 \%$

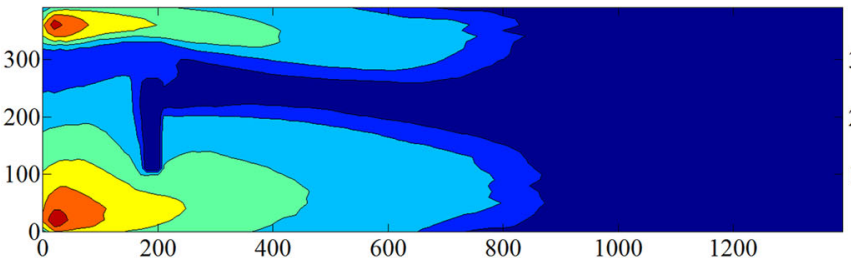

$\mathrm{t}=200$ days - Sweep Efficiency $=35.29 \%$

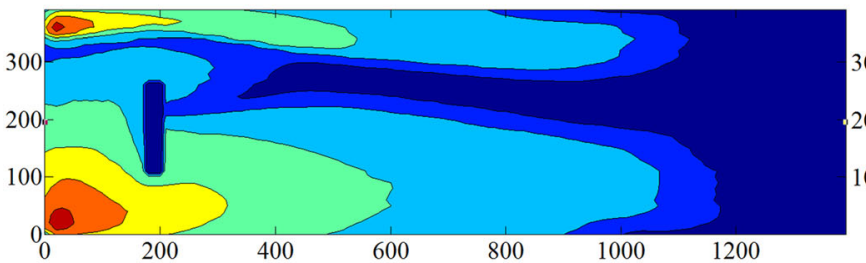

$\mathrm{t}=300$ days - Sweep Efficiency $=42.78 \%$

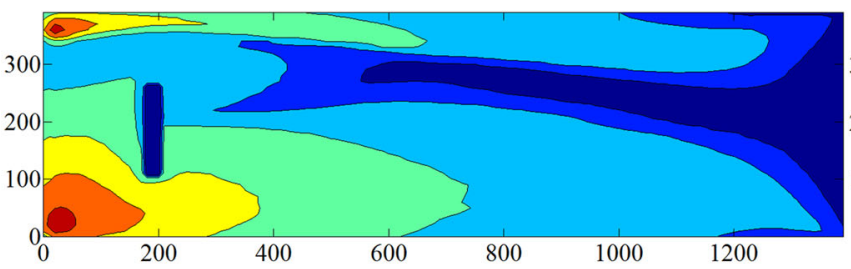

Breakthrough@ @t=467 days - Sweep Efficiency =46.88\%

\section{Base Case}

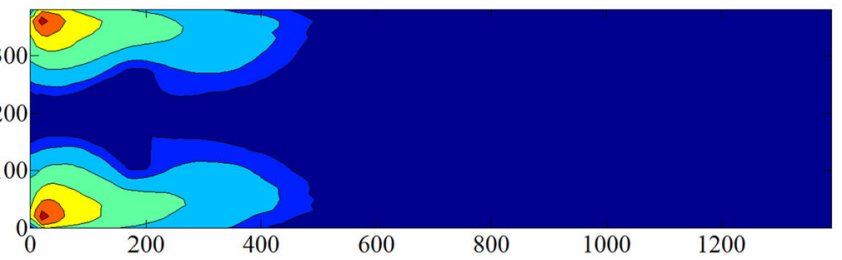

$\mathrm{t}=100$ days - Sweep Efficiency $=10.83 \%$

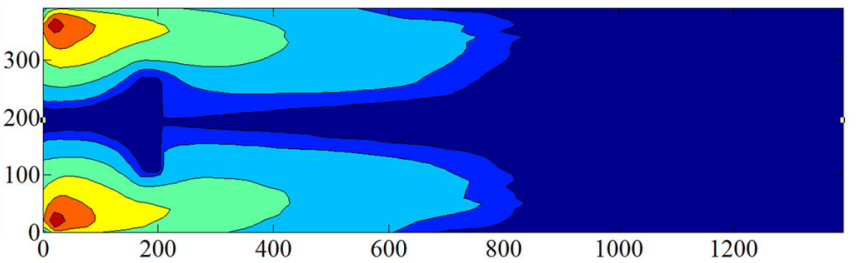

$\mathrm{t}=200$ days - Sweep Efficiency $=19.11 \%$

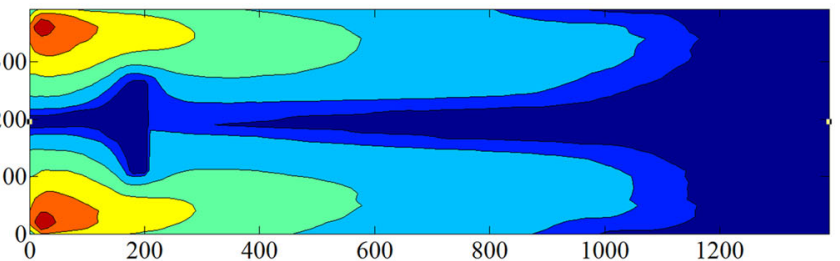

$\mathrm{t}=300$ days - Sweep Efficiency $=25.03 \%$

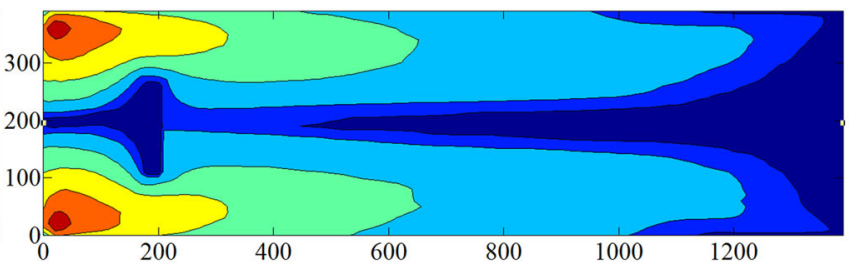

Breakthrough@t=353 days - Sweep Efficiency =27.57\%

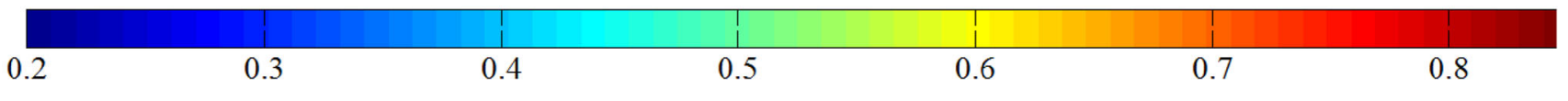

Fig. 5 Water saturation profile at different stages of the water flooding process (aspect ratio, $h_{2} / Y=0.375$, frequency of measurements, $f=15$ days, distance of obstacle, $d / X=2 / 14$ ) 
sweep efficiency $=100 \times \frac{\sum_{i=1}^{N}\left(S_{\mathrm{w}}^{i}-0.2\right)}{N \times(0.85-0.2)}$

where $S_{\mathrm{w}}^{i}$ is the water saturation in grid $i$ and $N$ is the total number of grid blocks located upstream of the obstruction. It is well noted that the employed optimization scheme not only significantly delays breakthrough, but also increases the sweep efficiency upstream of the obstruction by approximately $70 \%$. Figure 6 shows the required variations of the mean injection and production flow rates to achieve the shown optimized sweep efficiency.

\section{Sensitivity analysis}

To achieve the objectives of this investigation and study the effect of frequency of updates, aspect ratio of the obstacle, shape of the obstacle, and the distance between injection wells and the obstacle, on the sweep efficiency upstream of the obstacle various sensitivity analyses were conducted. In these studies, the various parameters were changed by a preset factor and their effect on the production optimization is reported in terms of percent sweep enhancement in the area of interest (i.e., upstream of the non-permeable obstacle).
Fig. 6 Optimal injection and production flow rates $\left(h_{2} / Y=0.375, f=15\right.$ days, $d / X=2 / 14$

Fig. 7 Sweep efficiency upstream of the obstacle as a function of the frequency of updates $\left(h_{2} / Y=0.375\right.$; $d / X=2 / 14)$
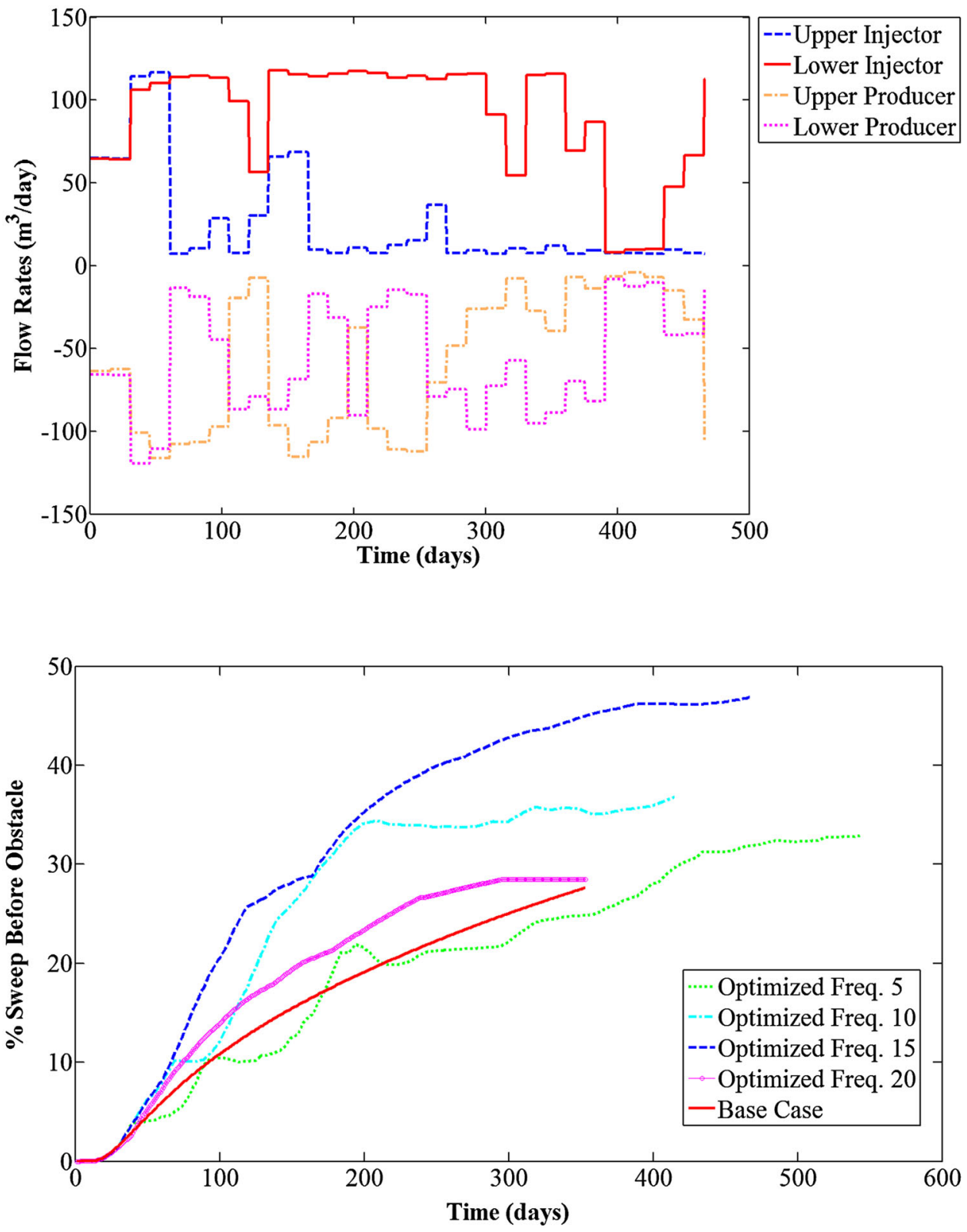


\section{Frequency of updates}

Changing the frequency of updates and thus allowing for forward propagation of the dynamical system has a direct impact on the efficacy of the presented optimization scheme. Figure 7 presents the percentage of sweep in the region upstream of the obstruction for various update frequencies. The results show that the frequency of the updates does not only affect the sweep efficiency but also

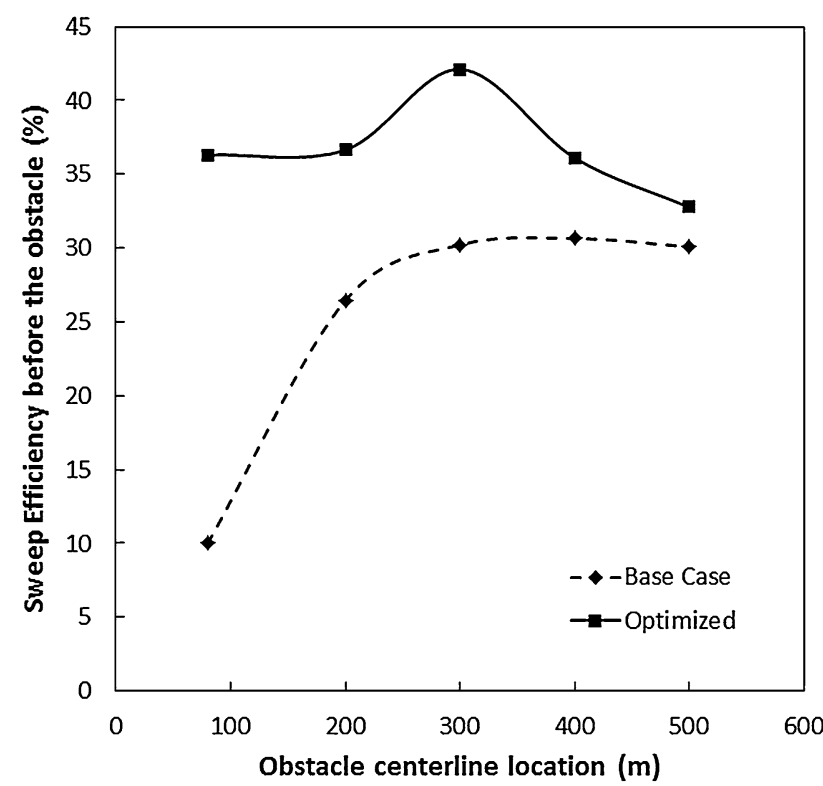

Fig. 8 Effect of the obstacle centerline location on sweep efficiency $\left(h_{2} / Y=0.25 ; f=15\right.$ days $)$

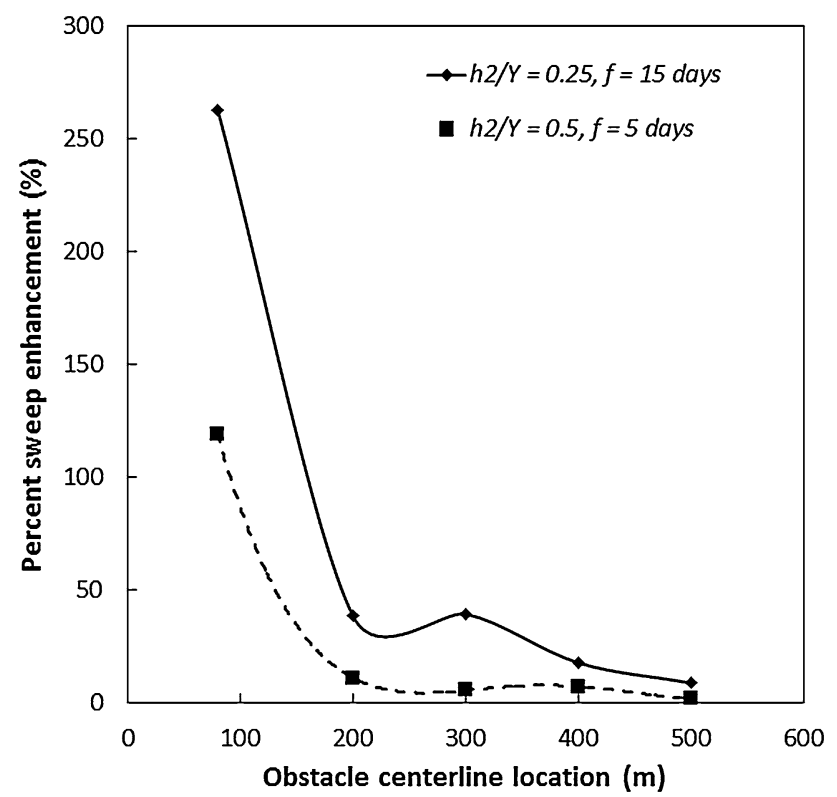

Fig. 9 Percent sweep enhancement when compared to the base case for two different conditions contributes to the delay or advancement of the breakthrough time. It is shown that the optimization efficiency will drop for both short and long update intervals (5 and 20 days, respectively). On the other hand significant sweep enhancement is noted for 10 and 15 days update frequencies. This is attributed to either not having sufficient time between updates to allow for the forward propagation of the dynamical system over few characteristic time scales or the lack of enough updates to significantly affect the model parameters and improve the sweep efficiency.

\section{Location of the obstacle}

The effect of the location of the obstacle with respect to the injection/production wells location has been studied by
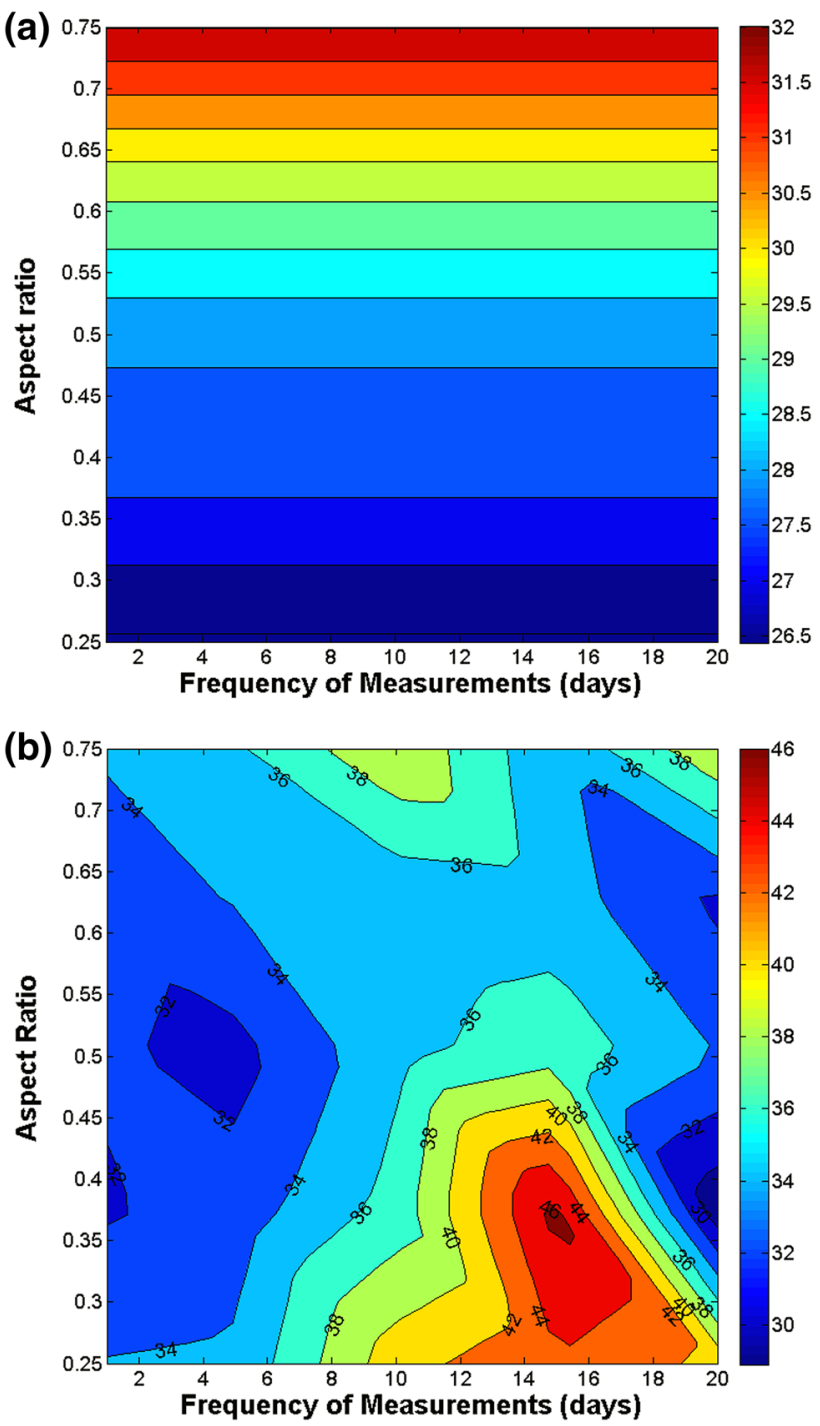

Fig. 10 Effect of varying the frequency of sampling and the aspect ratio on the percent sweep enhancement upstream of the obstacle a base case, $\mathbf{b}$ optimized $(d / X=2 / 14)$ 
varying the distance $d$. Various locations corresponding to $d=80, \quad 200, \quad 300, \quad 400, \quad$ and $\quad 500 \mathrm{~m}$ $(d / x=0.8 / 14,2 / 14,3 / 14,4 / 14,5 / 14)$, were considered. Figure 8 clearly shows that regardless of the obstacle location, the optimized solution has a better performance when compared to the base case. Furthermore, the base case situation, which corresponds to an equal volumetric flow rate injection, shows that the sweep efficiency increases as the distance between the obstacle and the injection points increases, and it plateaus at around $30 \%$. However, the optimized solution shows a local maxima at an obstacle location corresponding to $d / X=3 / 14$, where the sweep efficiency reaches $42 \%$ then starts gradually decreasing as the obstacle is moved farther.

\section{Optimized}
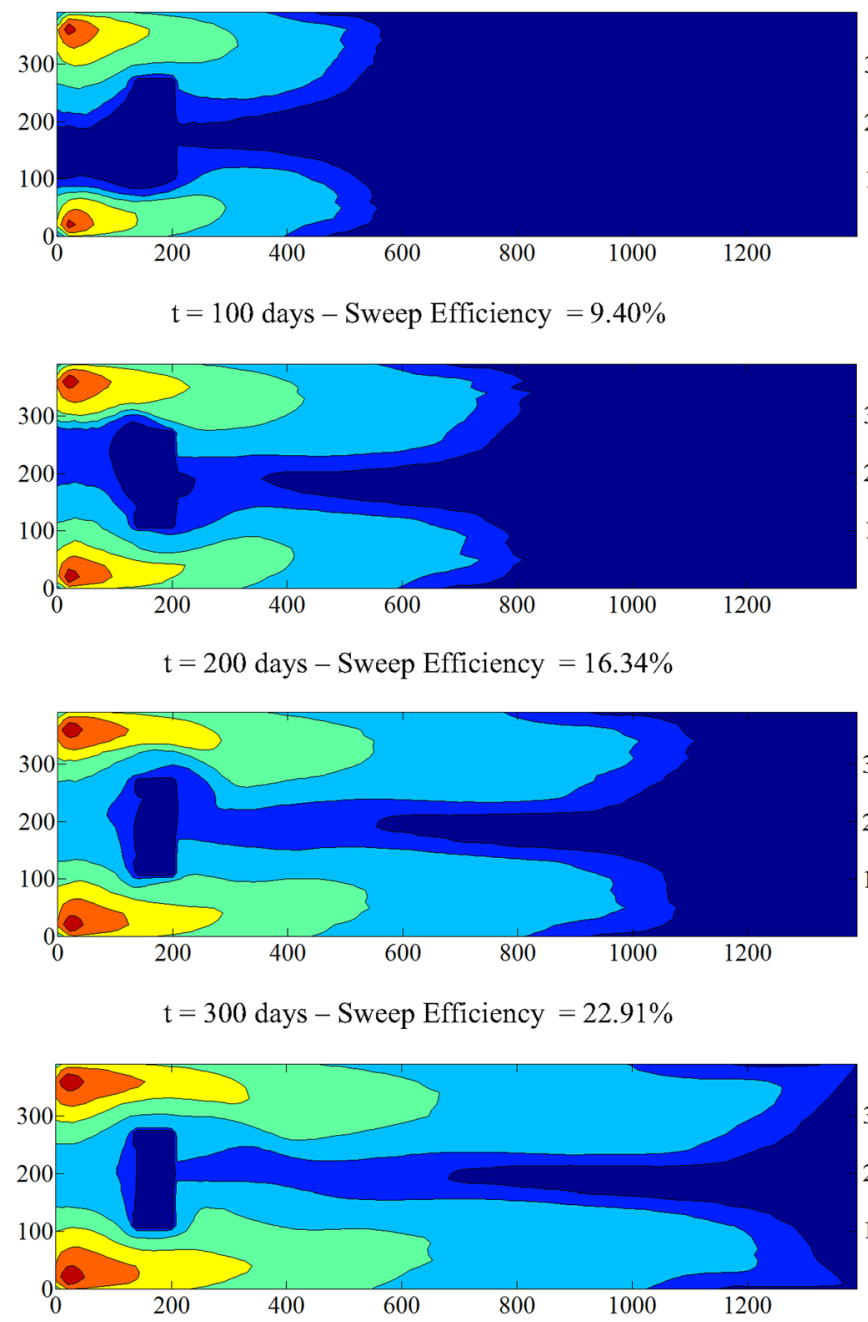

Breakthrough@t=367 days - Sweep Efficiency =24.79\%

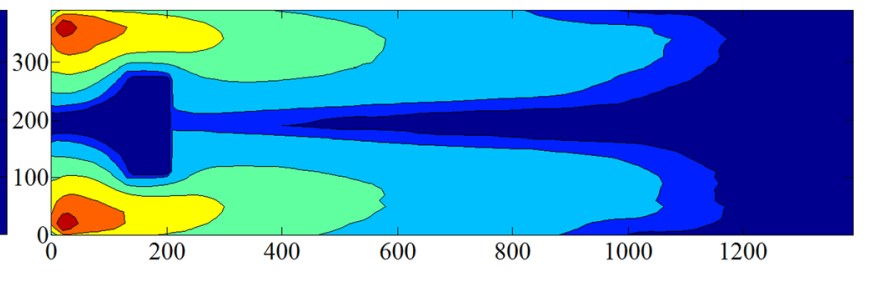

Further, the effect of the obstacle location while varying other model parameters, namely, the aspect ratio, $h_{2} / Y$, and the frequency of measurements, $f$, is presented in Fig. 9. This figure reports the information in terms of percentage sweep enhancement upstream of the obstacle when compared to the base case results, which were calculated according to Eq. (20).

Sweep enhancement $=100$

$$
\times\left(\frac{\text { sweep }_{\text {optimized }}-\text { sweep }_{\text {base case }}}{\text { sweep }_{\text {base case }}}\right)
$$

It is noticeable that the maximum enhancement is achieved when the obstacle is closer to the injection wells

\section{Base Case}
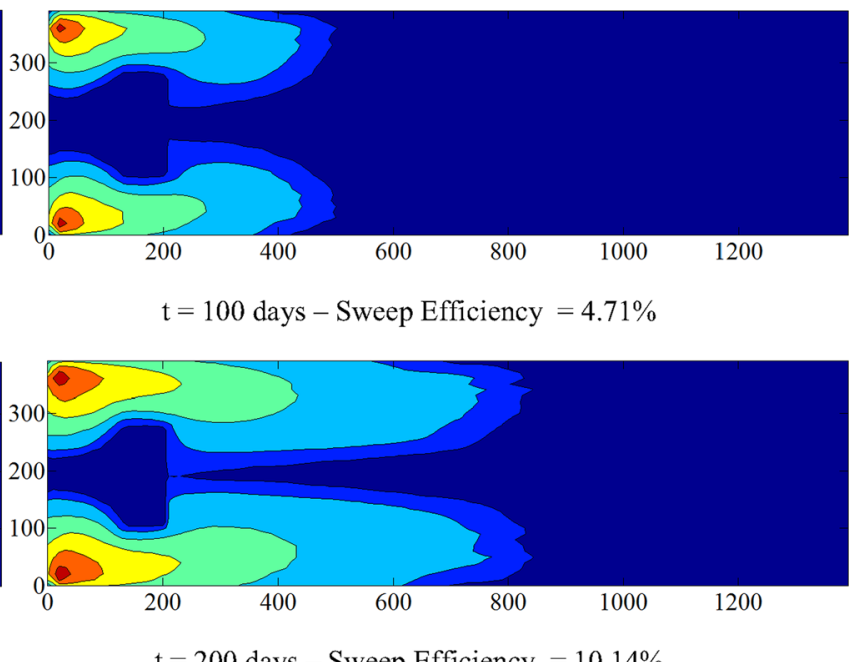

$\mathrm{t}=200$ days - Sweep Efficiency $=10.14 \%$

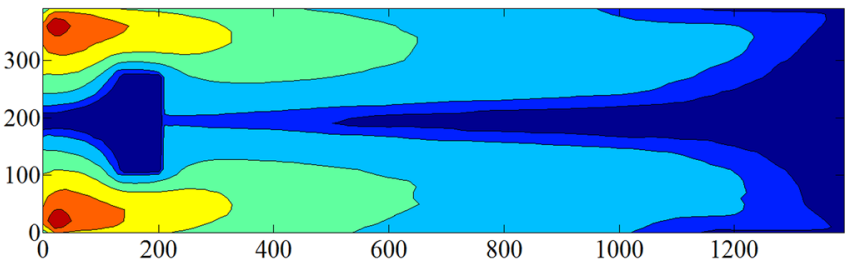

Breakthrough @ t=350 days - Sweep Efficiency =16.03\%

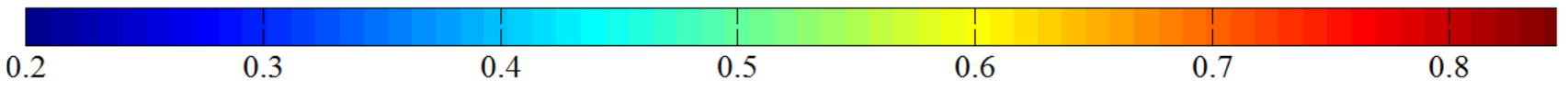

Fig. 11 Water saturation profile at different stages of the water flooding process for a U-shaped obstacle $\left(h_{2} / Y=0.4 ; f=15\right.$ days; $\left.d / X=2 / 14\right)$ 
with sweep enhancements as high as $260 \%$ could be achieved. However, the farther the obstacle is, the lower is the sweep enhancement and the lower is the effect of the various model parameters, namely, the aspect ratio and the frequency of updates.

\section{Aspect ratio of the obstacle}

The aspect ratio of the obstacle dictates how large the obstruction is with respect to the width of the reservoir. The larger the number is, the more constricted the flow will be, and consequently, the injected water will be trapped behind the obstacle rendering a higher sweep efficiency. Figure 10a and $\mathrm{b}$ are contour plots that show the effect of simultaneously changing the aspect ratio of the obstacle and the frequency of measurements on the sweep efficiency of the area upstream of the obstacle. Figure 10a clearly shows that irrelevant of the frequency of measurements, the sweep efficiency of the base case increases with the aspect ratio only. This is in line with the physical interpretation presented earlier. However, the situation is different when using the EnKF to optimize the flow. From Fig. 10b, the reader clearly discerns that the best case scenario would be for an aspect ratio, $h_{2} / Y=0.375$ and a frequency of measurements, $f=15$ days. Under those conditions, and for a rectangular shaped obstacle, it is expected that $46 \%$ of the area upstream of the obstacle be swept, compared to $\sim 28 \%$ for the base case.

\section{Shape of the obstacle}

The effect of changing the shape of the obstruction on the sweep efficiency was also considered in this work. When the shape is changed from rectangular to U-shaped, it is expected that the sweep efficiency upstream of the obstacle would be majorly affected because more oil would be trapped behind the boundaries of the obstacle. Therefore, when compared to a rectangular obstacle, the sweep efficiency is expected to be lower. Figure 11 presents snap shots of the water saturation profiles at different stages of the water flooding process. The snap shots correspond to the case with a U-shaped obstruction located at a distance $d=200 \mathrm{~m}$ and having an aspect ratio, $h_{2} / Y=0.4$. Furthermore, it is assumed that the edge of the U-shaped obstruction is extruding a distance, $h_{3}=60 \mathrm{~m}$ toward the location of the injectors. In the reported example, the optimization is carried out assuming that injection and production rates are updated at a frequency of 15 days. The sweep efficiencies in the region upstream of the obstruction are reported for both the optimized and base cases at different time stages leading to breakthrough. It is well noted that the employed optimization scheme enhances the sweep efficiency before the obstruction at breakthrough by approximately $55 \%$. Figure 12 also shows the required variations of the mean injection and production flow rates to achieve the shown optimized sweep efficiency.

\section{Conclusions}

This study presented the use of the EnKF for efficient CLRM. The objective was to control the injection and production rates to optimize the water flooding front dynamics in the presence of obstructions that might hinder the flow. This study is one of the very few available in
Fig. 12 Optimal injection and production flow rates for a U-shaped obstacle $\left(h_{2} / Y=0.4\right.$; $f=15$ days; $d / X=2 / 14$ )

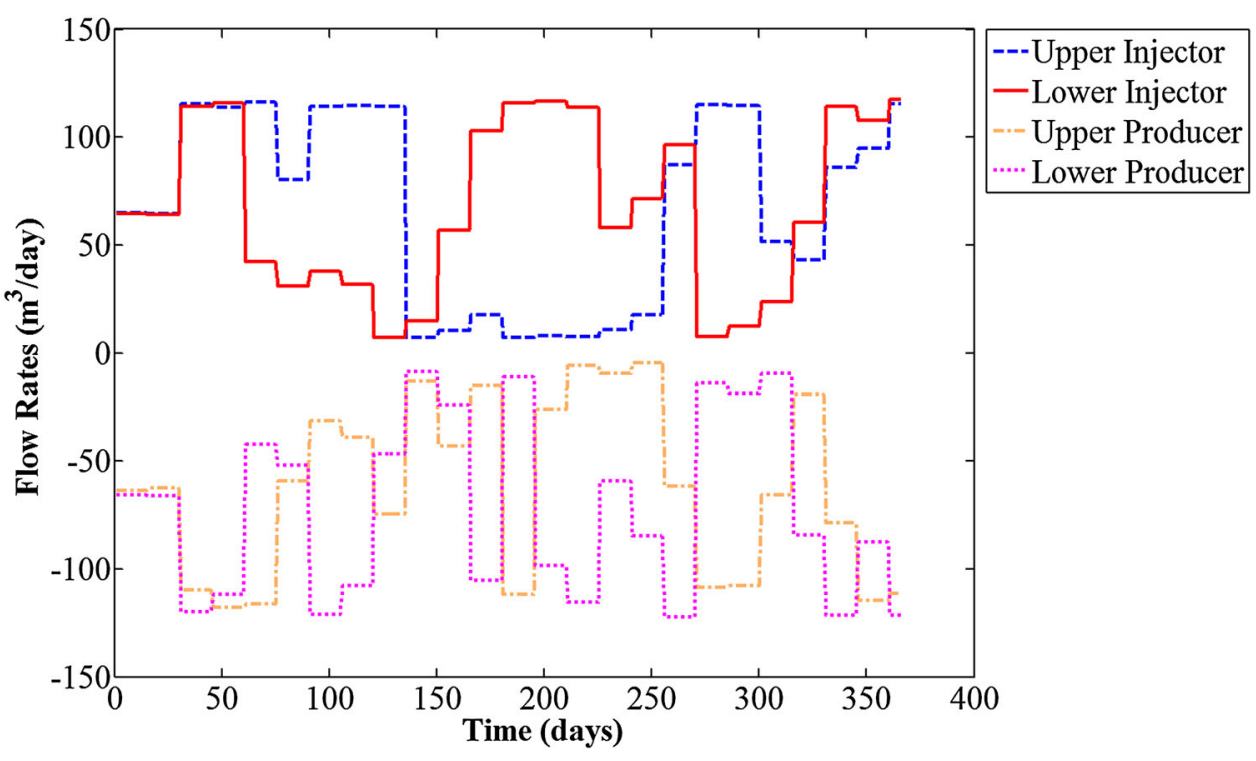


literature that considers the presence of an impermeable obstruction between the injectors and producers, and in contrast with previous studies that considered the use of EnKF for production optimization, this study investigated the effects of changing the frequency of updates, as well as changing the aspect ratio of the obstacle, shape of the obstacle, and the distance between injection/production wells and the obstacle, on the sweep efficiency behind the obstacle were presented. By changing these factors, and depending on the shape of the obstacle, it was found that the sweep efficiency upstream of an obstacle can be enhanced by up to $260 \%$ when compared to the base case of equal volumetric flow through the injectors.

Acknowledgments This study has been financially supported by the Munib and Angela Masri Institute of Energy and Natural Resources at the American University of Beirut.

Open Access This article is distributed under the terms of the Creative Commons Attribution 4.0 International License (http:// creativecommons.org/licenses/by/4.0/), which permits unrestricted use, distribution, and reproduction in any medium, provided you give appropriate credit to the original author(s) and the source, provide a link to the Creative Commons license, and indicate if changes were made.

\section{References}

Aanonsen SI, Nævdal G, Oliver DS, Reynolds AC, Vallès B (2009) The ensemble Kalman filter in reservoir engineering-a review. SPE J 14:393-412

Asadollahi M, Nævdal G, Dadashpour M, Kleppe J (2014) Production optimization using derivative free methods applied to Brugge field case. J Petrol Sci Eng 114:22-37

Asheim H (1988) Maximization of water sweep efficiency by controlling production and injection rates. SPE 18365

Bianco A, Cominelli A, Dovera L, Naevdal G, Valles B (2007) History matching and production forecast uncertainty by means of the ensemble Kalman filter: a real field application. In: EUROPEC/EAGE Conference and Exhibition, Society of Petroleum Engineers, London, UK, 11-14 June. SPE 107161

Brooks R, Corey A (1964) Hydraulic properties of porous media. Hydrology Papers 3
Brouwer D, Naevdal G, Jansen J, Vefring E, Van Kruijsdijk C (2004) Improved reservoir management through optimal control and continuous model updating. SPE 90149

Burgers G, Van Leeuwen PJ, Evensen G (1998) Analysis scheme in the ensemble Kalman filter. Mon Weather Rev 126:1719-1724

Chen Y, Oliver DS, Zhang D (2009) Data assimilation for nonlinear problems by ensemble Kalman filter with reparameterization. J Petrol Sci Eng 66:1-14

Dehdari V, Oliver DS (2012) Sequential quadratic programming for solving constrained production optimization - case study from Brugge field. SPE J 17:874-884

Evensen G (1994) Sequential data assimilation with a nonlinear quasi-geostrophic model using Monte Carlo methods to forecast error statistics. J Geophys Res Oceans 99:10143-10162

Evensen G (2003) The ensemble Kalman filter: theoretical formulation and practical implementation. Ocean Dyn 53:343-367

Fonseca R, Leeuwenburgh O, Van den Hof P, Jansen J (2014) Ensemble-based hierarchical multi-objective production optimization of smart wells. Comput Geosci 18:449-461

$\mathrm{Gu}$ Y, Oliver DS (2006) The ensemble Kalman filter for continuous updating of reservoir simulation models. J Energy Res Technol 128:79-87

Jansen J (2011) Adjoint-based optimization of multi-phase flow through porous media-a review. Comput Fluids 46:40-51

Kalman RE (1960) A new approach to linear filtering and prediction problems. J Basic Eng 82:35-45

Krymskaya M, Hanea R, Verlaan M (2009) An iterative ensemble Kalman filter for reservoir engineering applications. Comput Geosci 13:235-244

Lorentzen RJ, Berg AM, Nævdal G, Vefring EH (2006) A new approach for dynamic optimization of water flooding problems. Paper SPE 99690

Nævdal G, Brouwer DR, Jansen J (2006) Waterflooding using closedloop control. Comput Geosci 10:37-60

Nwaozo J (2006) Dynamic optimization of a water flood reservoir. MS dissertation

Oliver DS, Chen Y (2011) Recent progress on reservoir history matching: a review. Comput Geosci 15:185-221

Saad G, Ghanem R (2009) Characterization of reservoir simulation models using a polynomial chaos-based ensemble Kalman filter. Water Resour Res 45

Sarma P, Durlofsky LJ, Aziz K, Chen WH (2006) Efficient real-time reservoir management using adjoint-based optimal control and model updating. Comput Geosci 10:3-36

van Essen G, Zandvliet M, Van den Hof P, Bosgra O, Jansen J (2009) Robust waterflooding optimization of multiple geological scenarios. SPE J 14:202-210

Wang C, Li G, Reynolds AC (2009) Production optimization in closed-loop reservoir management. SPE J 14:506-523 